\title{
Internet de las cosas en el tratamiento de variables Agro-climatológicas en huertos urbanos
}

\author{
Nancy Esperanza Olarte López \\ http://orcid.org/0000-0001-7029-4006 \\ Universidad Militar Nueva Granada, Colombia \\ David Enrique Torres Rojas ${ }^{2}$ \\ https://orcid.org/0000-0003-2119-0213 \\ Universidad Militar Nueva Granada, Colombia \\ Roland Fernando Franco Ramos ${ }^{3}$ \\ https://orcid.org/0000-0002-8031-6181 \\ Universidad Militar Nueva Granada, Colombia
}

Internet of things in the treatment of Agro-climatological variables in urban orchards

Recibido:04-02-2021

Aceptado: 27-04-2021

\section{Cita Recomendada}

Olarte, N. E., Torres, D. E. y Franco, R.F. (2021). Internet de las cosas en el tratamiento de variables agro-climatológicas en huertos urbanos. Hamut'ay, 8(1), 33-41.

http://dx.doi.org/10.21503/hamu.v8i1.2234

\begin{abstract}
Resumen
El almacenamiento de datos contribuye a la predicción de posibles comportamientos en los cultivos como: sequías, heladas, lluvias constantes, plagas, aumento de la radiación solar entre otros. Incluso para la contingencia actual por medidas de aislamiento preventivo a causa del Covid, el control de datos almacenados es aún más necesario debido a que se disminuye la presencia in situ en función de la toma de datos. De hecho, algunas personas han optado por tener huertos urbanos desde casa a causa de la cuarentena, y otros por economía para obtener sus propios insumos consumibles.

El tratamiento de variables agro-climatológicas en huertos urbanos se realizó como proyecto de iniciación científica durante el año 2020, incluso, el desarrollo de sus objetivos se llevó a cabo bajo la pandemia y fue financiado por la Universidad Militar Nueva Granada en Colombia.

Como consecuencia se podría considerar desde locaciones cercanas rurales o urbanas la implementación de técnicas bajo IoT (Internet de las Cosas) que garanticen en corto plazo la producción a pequeña y mediana escala de alimentos necesarios para la canasta familiar.
\end{abstract}

Palabras Clave: Agro-climatológica, Almacenamiento, Huerto, IoT, Internet de las Cosas.

\footnotetext{
1. Grupo de Investigación e innovación Tecnológica en Electrónica y Comunicaciones (GI-iTEC) / Semillero de Investigación Faraday; Magister en Tecnologías de la Información Aplicadas a la Educación; Especialista en Tecnologías de la Información Aplicadas a la Educación; Ingeniera en Telecomunicaciones; docente planta tiempo completo programa de Tecnología en Electrónica y Comunicaciones, Facultad de Ingeniería Universidad Militar Nueva Granada, Colombia; nancy.olarte@unimilitar.edu.co

2. Grupo de Investigación e innovación Tecnológica en Electrónica y Comunicaciones (GI-iTEC) / Semillero de Investigación Faraday; egresado programa de Tecnología en Electrónica y Comunicaciones, Facultad de Ingeniería Universidad Militar Nueva Granada, Colombia; davidtorresr96@gmail. com

3. Grupo de Investigación e innovación Tecnológica en Electrónica y Comunicaciones (GI-iTEC) / Semillero de Investigación Faraday; estudiante IV semestre programa de Tecnología en Electrónica y Comunicaciones, Facultad de Ingeniería Universidad Militar Nueva Granada, Colombia, rolandfranco97@gmail.com
} 


\begin{abstract}
Data storage contributes to the prediction of possible crop behaviors such as: drought, frost, constant rainfall, pests, increased solar radiation, among others.

Even for the current contingency of preventive isolation measures due to Covid-19, the control of stored data is even more necessary due to the fact that the presence on site is reduced in function of data collection. In fact, some people have chosen to have urban gardens from home because of the quarantine, and others for economy to obtain their own consumable inputs.

The treatment of agro-climatological variables in urban orchards was carried out as a scientific initiation project during the year 2020, even, the development of its objectives was carried out under the pandemic and was financed by the Universidad Militar Nueva Granada in Colombia.

As a consequence, the implementation of techniques under IoT (Internet of Things) could be considered from nearby rural or urban locations to ensure in the short term the small and medium scale production of food necessary for the family basket.
\end{abstract}

Key words: Agro-climatological, Storage, Orchard, IoT, Internet of Things.

\section{Introducción}

Una diversidad de empresas y organizaciones se han visto obligadas a tener que utilizar el Internet de las Cosas (IoT) en mejoras de sus procesos, ya que este es transversal a muchos usos automatizados en granjas, agricultura inteligente, de precisión, industrial, intensiva (Sánchez y Restrepo, 2018), en cultivos de hortalizas en campo, urbanos y cultivos hidropónicos. También este monitoreo aplica hacia huertos comunitarios de tipo vertical (Algert, Baameur \& Renvall, 2014), para un mejor control en la optimización de espacios, distribución de sensores y disminución de costos. Las granjas verticales (Ver Fig. 1) son una de las nuevas estrategias para la producción agrícola, ayudan a optimizar espacios y maximizar los volúmenes de la cosecha dentro de ambientes controlados (Diaz, 2016). Para sistemas de control de riego, incluso para aquellas granjas verticales modulares (Ramírez, 2020), se deben tener en cuenta variables tales como: viento, temperatura, humedad de suelo, ambiente y luminosidad, esta última puede ser también mediante luz artificial si la natural es mínima; además de la inclusión de actuadores que permiten la automatización en el riego e iluminación que permitan a la planta mantener sus condiciones ideales de crecimiento ambiente (Martínez y Pérez, 2017).

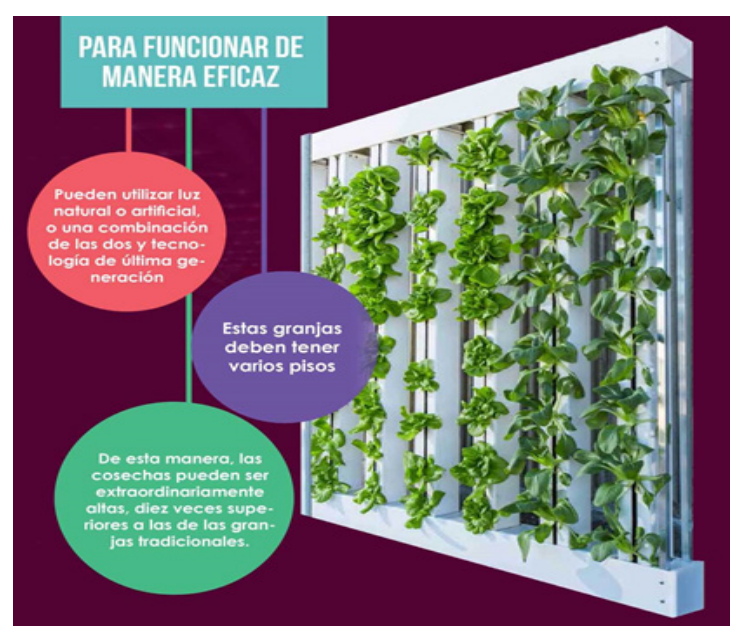

Figura 1. Granjas Verticales y sus consideraciones (TEC, 2020)

Entre las variables que se pueden monitorear se encuentra el riego, tipo de tierra, el clima, las plagas y la fertilización. Así mismo es indispensable mejorar factores como: tener una alta conectividad a la red para tener una comunicación síncrona hacia el monitoreo mediante dispositivos IoT, la capacidad de procesamiento de datos ya que muchos de los sensores y actuadores que se utilizan tienen una capacidad de almacenamiento muy baja y es necesaria la vinculación entre el internet, los dispositivos IoT y la nube. Así mismo, aumentar la potencia energética debido a que algunos sensores trabajan sin fuente de alimentación propia, otro aspecto es la gobernanza de datos, seguridad y privacidad para el acceso 
y control privado del agricultor (Orozco et al., 2018).

Este tipo de aplicaciones para el monitoreo automatizado de cultivos urbanos o también en pequeños invernaderos de forma local son indispensables en Colombia debido a la fuerte variación de los climas que afectan los cultivos y que perjudican su producción, ocasionando algunas pérdidas económicas al sector agropecuario.

A partir de los avances de las recientes investigaciones en el mundo, es importante resaltar la posibilidad de realizar iniciativas que permitan de manera local monitorear huertos urbanos (Sanz-Cobeña, et al, 2017), por lo anterior, se tiene como objetivo general: identificar los métodos de monitoreo de invernaderos, incluyendo almacenamiento de datos y procesamiento para su posterior análisis. Y como objetivos específicos: i. Conocer la optimización de los sensores al obtener medidas de cultivos frente a los diferentes factores dentro de un invernadero, y ii. Almacenar y procesar los datos obtenidos de los sensores en función de las condiciones del invernadero.

\section{Internet de las Cosas en huertos urbanos}

Según Tsiatsis et al., (2018) al Internet de las Cosas (IoT) se define como un dominio que integra diferentes tecnologías y campos sociales. Son sistemas que pueden ser de pequeña escala hasta escala global. También puede definirse al IoT como la interconexión de diferentes dispositivos y objetos por medio de una red privada o Internet, de manera que pueden interactuar y ser manejados desde un servidor. Los dispositivos van desde sensores y elementos mecánicos hasta objetos cotidianos como pueden ser la nevera o la ropa. Las cosas interactúan sin necesidad de la intervención humana, en lo que se conoce como interacción de máquina a máquina o M2M (machine to machine).

Con relación a IoT en huertos urbanos, investigadores en Corea han construido un sistema de granja inteligente (Yoon et. al., 2018) que combina módulos de comunicación Bluetooth de baja potencia y redes de área amplia de baja potencia (LPWAN), incluida la red de comunicación por cable utilizada en la granja que permite solucionar problemas de energía y limitaciones de espacio, además de implementar el método de comunicación MQ Telemetry Transport (MQTT), que es un protocolo dedicado de IoT para el control de variables, lo que convierte a todo el sistema en nodo - servidor - base de datos y estructura de control externo, con la finalidad de que el factor sea de alta conectividad.

Este mismo método de comunicación (MQTT junto con LPWAN) ha sido utilizado en India, pero con la diferencia de implementar LoRa, una de las nuevas tecnologías LPWAN cuenta con grandes ventajas para proyectos de IoT, con dispositivos alimentados por baterías (Puengsungwan \& Jiraserccamomkul, 2018). También ha sido empleado de manera exitosa para la comunicación de los datos de temperatura externa de los viñedos para garantizar la calidad óptima en la producción de uva para vino (Kodali, Yerroju \& Sahu, 2018).

De hecho, LoRa ha sido implementada en investigaciones en Corea del Sur (Davcev et. al., 2018), sin embargo, ha tenido algunas desventajas como: la transferencia de datos multimedia debido a su baja velocidad y ancho de banda, no obstante, esto se puede corregir con la propuesta de Mookeun y sus colaboradores, quienes recomiendan dividir una imagen y enviar solo el marco seleccionado o área de interés para disminuir los datos a transferir (Ji et al., 2019).

Paralelamente también se ha venido trabajando en otro tipo de redes como las WSAN o redes de área inalámbrica de almacenamiento, las cuales permiten recolectar los datos obtenidos de los puntos monitoreados al mismo tiempo, escalables hacia un conjunto de campos o espacios de difícil acceso y control mediante la red guiada (López, Chávez y Sánchez, 2017), disminuyendo así costos en materiales, mantenimiento y tendidos de cable.

La gran cantidad de información registrada, a partir de la adquisición de datos de los sensores, lleva a la necesidad de pensar en cómo administrar de una manera eficiente su procesamiento. Con respecto al almacenamiento, mantenimiento y análisis de las enormes cantidades de datos 
generados por los dispositivos IoT, Cloud Computing y Fog Computing han contribuido como soluciones suficientes (Tzounis et al., 2017), la primera agrupa datos en la nube, mientras la segunda permite almacenar datos obtenidos en los mismos dispositivos permitiendo su interoperabilidad, pero también se convierte en un desafío lograr su seguridad frente a ataques externos.

Recientes investigaciones han decidido trabajar de manera descentralizada la cantidad de información obtenida de los sensores fijos y móviles en tiempo real, lo anterior en un eventual suceso si la nube llega a fallar o a tener un ataque externo, permitiendo enviar datos desde Unidades de Telemetría Remota (RTU) (Suciu, Istrate \& Dițu, 2019) quienes serían los nodos hacia el servidor bajo una comunicación con topología en malla y no centralizada, posibilitando en un ataque que fallen uno o varios nodos, pero encontrando caminos alternos para no fallar la comunicación síncrona.

De hecho, algunas aplicaciones han sido desarrolladas para reconocer a tiempo el ciclo de vida de las hortalizas, un caso particular fue en Tailandia, donde investigadores de la Universidad Tecnológica de Thonburi detectaron el estrés temprano en lechugas, es decir, predijeron si por altas temperaturas u otros agentes como el nitrógeno, la hortaliza perdía agua por periodos de sequía o sufrir quemaduras por los rayos solares. Los resultados de este estudio permitieron que en tiempo real el administrador de la granja, el experto y el propietario esté en constante comunicación para el control, monitoreo y toma de decisiones a través de los datos adquiridos por el sensor de fluorescencia de clorofila (CHF), no obstante, al incluir en el sistema de detección la función de estrés ambiental y la función de estrés de la hoja, la precisión del sistema mejora (Puengsungwan \& Jiraserccamomkul, 2018). El diagrama del sistema implementado se puede apreciar en la siguiente Figura 2, fuente de los autores como diseño en el año 2018.

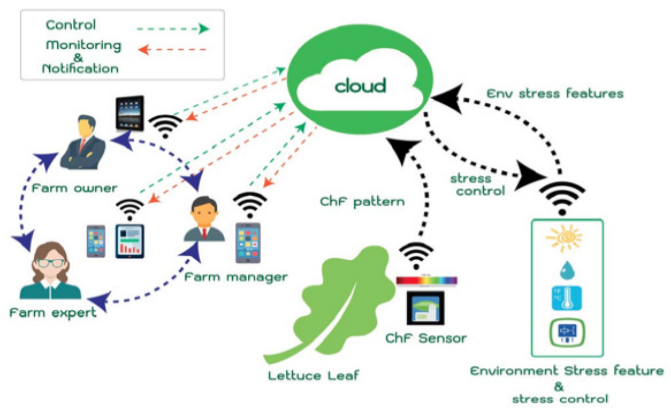

Figura 2. Propuesta de IoT basado en el sistema de detección de estrés.

Fuente: (Puengsungwan y Jiraserccamomkul, 2018)

Se destaca también, la gran importancia de reconocer los impactos que se pueden lograr involucrando este tipo de investigaciones y sus contribuciones hacia huertos urbanos a nivel nacional, como se mencionan a continuación:

Ambiental. Con la identificación del uso adecuado de sensores se puede disminuir el uso excesivo de agua en el riego, también al controlar factores ambientales como humedad y temperatura se puede detectar a tiempo la fatiga o estrés de las plantas.

Salud humana. A corto plazo, al tener un control adecuado de los cultivos, se puede identificar a tiempo cuáles frutos o vegetales consumibles por los seres humanos se encuentran en óptimas condiciones, también se podría predecir el tiempo de vida de cada tipo de cultivo.

Seres vivos. A largo plazo pueden existir modelos de proyección de cultivos quienes mediante un sistema de control local de hortalizas pretenden integrar la agricultura de invernadero a la infraestructura energética como solución alimentaria.

Económico. A largo plazo pueden existir modelos de proyección de cultivos quienes mediante un sistema de control local de hortalizas pretenden integrar la agricultura de invernadero a la infraestructura energética como solución alimentaria.

Social. Incrementa la interacción de la población con las Tecnologías de la Información y las Comunicaciones, promueve en la población el debido uso de recursos para el cuidado de cultivos. Tecnológico. Desde la perspectiva tecnológica, almacenar datos y procesarlos ayuda a tener un 
uso más eficiente de recursos para el tiempo de respuesta en las tomas de decisiones frente al control de cultivos urbanos y también invernaderos

Almacenamiento de datos. Dentro de la agricultura inteligente, contribuye a la predicción de posibles comportamientos en los cultivos como: sequías, heladas, lluvias constantes, plagas, aumento de la radiación solar entre otros.

Así mismo, en la actualidad es necesario contar con sistemas para almacenar información a gran escala, necesitando medir continuamente una variable, y esta se almacena a través de los datos adquiridos de los sensores del proceso. Como ejemplos, se tienen, las imágenes de las predicciones meteorológicas o las visualizaciones de tráfico en tiempo real en las plataformas como Google Maps (Zuil, 2015) (Ver Figura 3) o Waze para calcular la ruta más rápida al destino.

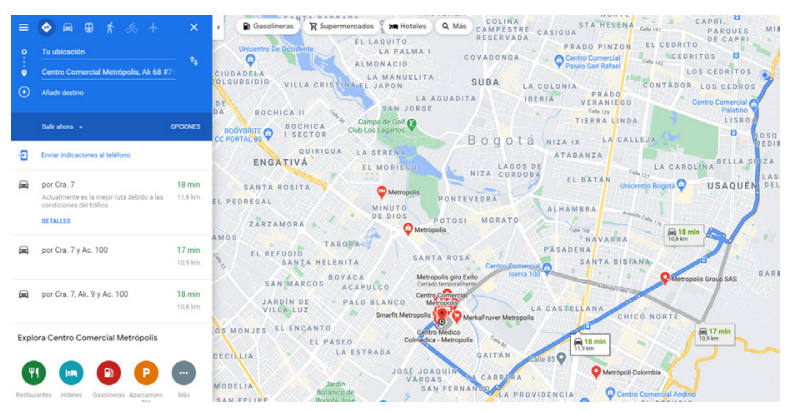

Figura 3. Cálculo de ruta a destino.

Fuente: https://www.google.com/maps

Los algoritmos para estas predicciones y control en tiempo real podrían instalarse también en los sistemas de almacenamiento de datos para el monitoreo de huertos urbanos en mediano plazo.

\section{Métodos y Procedimiento.}

\section{Participantes}

Las muestras estuvieron constituidas por hortalizas de tipo fruto: pimentón, tipo bulbo: cebolla, tipo hojas: lechuga y tipo raíces: zanahoria, controladas en un ambiente al interior de la vivienda de uno de los autores del proyecto, ubicada al sur de la ciudad de Bogotá, dentro de un pequeño patio interno, en el cual se pudo tener un control de variables como luminosidad y humedad, bajo controles manuales iniciales diarios, y luego de dos meses cada tercer día, teniendo en cuenta la recolección local de datos obtenidos de sensores, el tratamiento de los datos y su procesamiento para posterior transmisión local.

Los sensores utilizados fueron adquiridos en almacenes de componentes electrónicos, algunos en línea debido al cierre comercial de algunos establecimientos a causa de la pandemia, no obstante, fueron escogidos aquellos cuyas características fueran: fácil adquisición, bajo costo, precisión alta en los datos obtenidos y de vital importancia, que fuesen compatibles con la plataforma Arduino, los tipos de sensores seleccionados se podrán apreciar en los resultados de este artículo.

\section{Procedimiento}

Se realizó un estudio secuencial y experimental, con corte o prioridad cualitativa en los primeros dos meses mediante la adquisición y el análisis de datos, por otro lado, los tres meses siguientes contaron con una implementación cuantitativa que integra la interpretación de datos, el almacenamiento y acceso de estos en la nube.

A partir de los objetivos planteados, a continuación, se presentan la metodología utilizada para el proyecto, la cual se llevó a cabo en cuatro fases (Ver Figura 4):
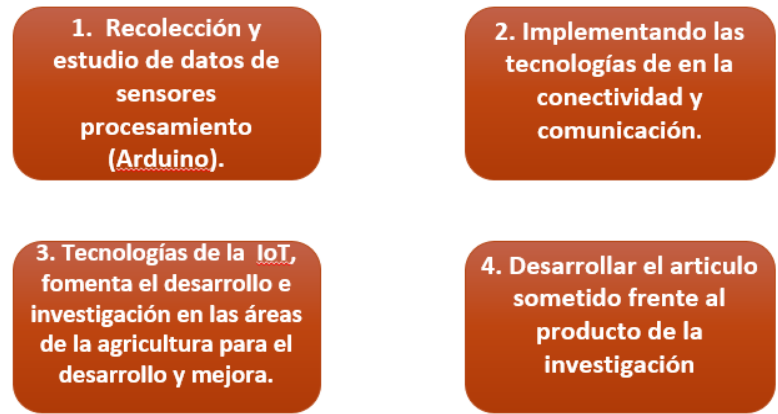

Figura 4. Fases del proyecto de investigación. Fuente: elaboración propia (2021)

Para el desarrollo de las fases se tuvieron en cuenta las siguientes actividades (Ver Figura 5): elección de sensores, identificación de medios de comunicación y selección de tarjetas o dispositivos para procesamiento y visualización de datos. 

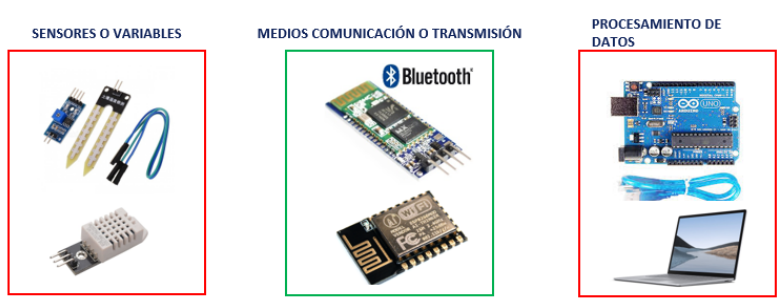

Figura 5. Actividades relacionadas con las fases del proyecto. Fuente: Elaboración propia (2021)

\section{Resultados}

A partir de los objetivos planteados y actividades realizadas, a continuación, se presentan los diferentes resultados obtenidos:

\section{Caracterización de Sensores}

Para los sensores, se caracterizaron aquellos que proporcionaran la extracción de datos que pudiesen ser digitalizados, para posteriormente, ser almacenados como: la humedad del suelo, luz ambiental y humedad del ambiente, factores claves en el control de un huerto urbano (Ver Figura 6).

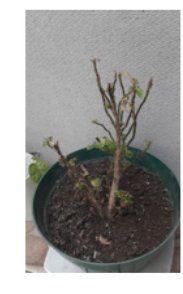

Humedad del suelo

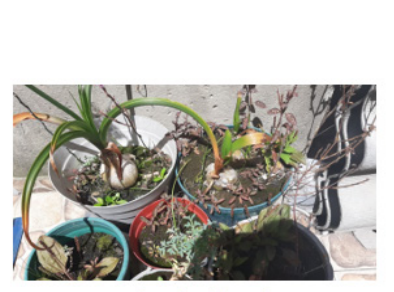

Luz Ambiental

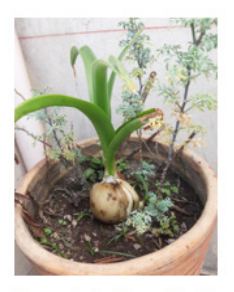

Humedad del ambiente
Figura 6. Variables características para cada sensor.

Fuente: Elaboración propia (2021)

En cuanto al sensor de humedad de suelo, se escogió el denominado higrómetro de tierra (Módulo YL-69) con sonda, salida digital y análoga (Ver Figura 7 a.), así mismo, para conocer la humedad del ambiente se escogió el sensor de precisión DHT-22, el cual también proporciona datos de humedad (Ver Figura 7 b.), y por último el sensor de luz de ambiente digital BH750 con valores de medida en Lux (Ver Figura 7 c.). Los tres con entradas de alimentaciones mínimas de voltaje y compatibles e integrables al módulo de Arduino.
Así mismo, fue importante revisar factores de luminosidad, temperatura ambiente, suelo fértil y medidas para acomodar espacios de diferentes tipos de cultivos de hortalizas y posibles conexiones para acceso local y remoto, identificando posibles técnicas automatizadas para riego y almacenamiento de variables.

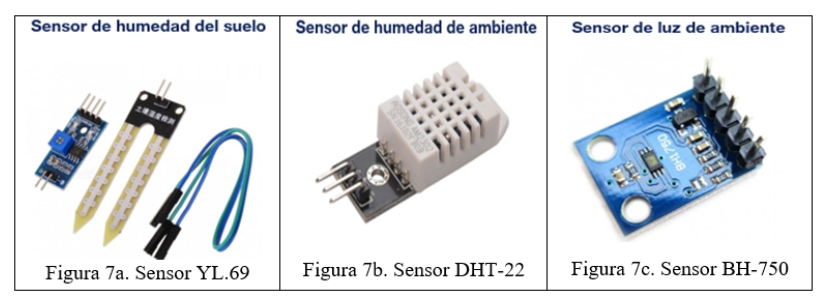

Figura 7. Sensores seleccionados

Fuente: Digi-Key Electronics

\section{Módulos de comunicación}

Para la comunicación local de las variables agro-climatológicas del huerto urbano, se escogieron tres tecnologías cuyos dispositivos son comerciales y compatibles con dispositivos móviles como computadores, pantallas LCD y celulares, entre estos dispositivos se tuvieron en cuenta: Módulo Bluetooth HC06 (Ver Figura 8 a.), módulo Wifi SERIAL WSP8266 integrado hacia computadora (Ver Figura 8 b.), y finalmente el módulo GPRS-GSM SIM800L para mensajes de texto hacia celular (Ver Figura 8 c.).

\begin{tabular}{|c|c|c|}
\hline Modulo Bluetooth & Modulo Wifi & Modulo GSM \\
Figura 8 a. Módulo HC06 & $\begin{array}{c}\text { Figura 8b. Módulo } \\
\text { WSP8266 }\end{array}$ & Figura 8c. Módulo SIM800L \\
\hline
\end{tabular}

Figura 8. Módulos de comunicación de datos Fuente: Digi-Key Electronics

Procesamiento y almacenamiento de variables En cuanto al procesamiento de los datos, se escogió el módulo de Arduino compatible con varias entradas digitales de los sensores, con el fin de unificar los datos extraídos y ser enviados en un único formato que permitiera ser leído y almacenado en la nube para visualización y tratamiento de datos desde dispositivos como computadores o portátiles posterior a su transmisión o envió mediante la red inalámbrica. (Ver Figura 9). 


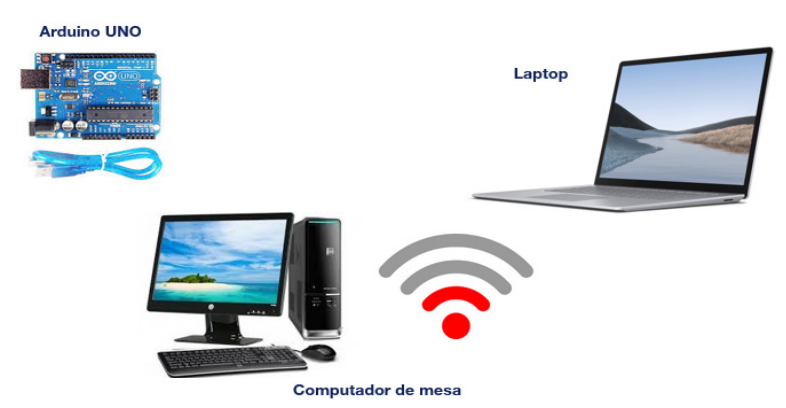

Figura 9. Procesamiento de datos en dispositivos. Fuente: Elaboración propia (2021)

\section{Estudios de secuencia y procesamiento}

$\mathrm{Al}$ almacenar datos en la nube, es posible realizar un seguimiento más eficiente in situ o local en función del monitoreo del cultivo urbano, se guardaban datos según el día y hora tomados para cada tipo de hortaliza cultivada, esto permitió considerar diferentes comportamientos de cada cultivo a lo largo del tiempo, y cada cuanto se podría predecir condiciones como riego, temperatura y luminosidad (Ver Figura 10).
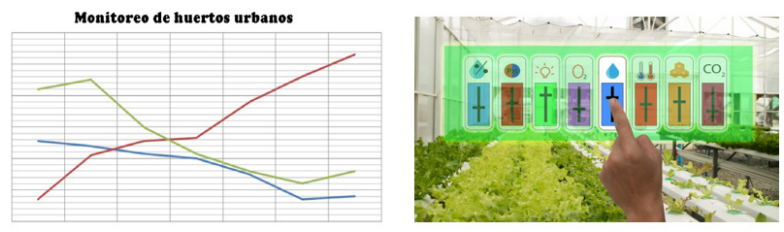

Figura 10. Secuencia de comportamientos. Fuente: Elaboración propia (2021)

En cuanto a la identificación de sensores, durante las medidas de aislamiento preventivo derivadas de la cuarentena por Covid 19, fue necesario adquirirlos mediante compras por internet y el tiempo de ejecución del proyecto se prolongó dos meses más, al ser sensores que fuesen compatibles con Arduino, son especiales para su búsqueda por internet.

Así mismo, por el momento se tienen almacenamientos locales de información, pero se proyecta no sólo continuar con el monitoreo de variables sino la automatización de actuadores para el riego continuo y verificación de luminosidad, humedad y temperatura, lo que a mediano plazo promueve el diseño de alarmas en el sistema, tratados en tiempo real desde el usuario mediante su dispositivo móvil (Ver Figura 11).

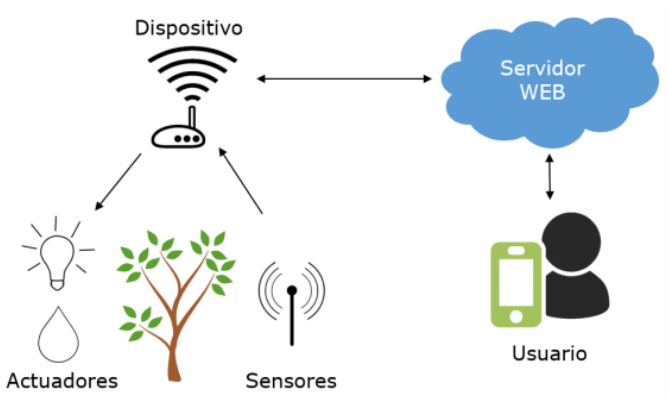

Figura 11. Modelo proyectado a mediano plazo. Fuente: (Rincón, 2015)

A mediano plazo se podrían instrumentar redes neuronales para cada tipo de hortaliza, lo anterior en función de predecir fatiga o estrés causados por exceso de luminosidad, humedad relativa, riego o temperatura excesiva.

\section{Discusión y Conclusiones}

De los resultados anteriores, los autores en la discusión plantean que, aunque no se puede desconocer la importancia del uso de sensores para obtener datos precisos en el monitoreo de temperatura, humedad relativa y luminosidad (incluso para tener resultados al instante) obtener los sensores que sean adaptables a nuevas tecnologías de comunicaciones sigue siendo costoso frente a aquellos que solo toman una muestra visual sin que se registren datos para su posterior almacenamiento.

Incluso, en momentos como la actual pandemia es de vital importancia tener un mecanismo de monitoreo al alcance para los huertos urbanos, sin necesidad de tener un riesgo por exposición al tener cultivos externos (Chipantiza-Masabanda, Bonilla-Bonilla \& Jativa-Reyes, 2021).

Llevar a cabo cultivos en invernaderos urbanos promueve tener a la mano productos básicos de la canasta familiar, incidiendo en la economía de los hogares para autoabastecerse incluso en momentos de aislamiento preventivo por pandemias; esto permite a su vez, no correr riesgos de ser afectados por un virus al salir de hogar para adquirir los productos y de otra forma, el tener autonomía en el control ayuda a que cada vez se tenga eficacia en el crecimiento de cada producto. 
Monitorear variables de forma local, ayuda también a que se logre en corto plazo incluir mayor número de hortalizas (dependiendo del tamaño de la locación) o proyectar este control hacia invernaderos medianos, obviamente incrementando el tamaño de datos almacenados en el servidor o en la nube.

\section{Agradecimientos}

A la Universidad Militar Nueva Granada (UMNG) por el financiamiento del proyecto PIC-ING-3242: Almacenamiento de variables agro-climatológicas en huertos urbanos. Al grupo de investigación GI-iTEC y al semillero Faraday del programa de Tecnología en Electrónica y Comunicaciones.

\section{Referencias bibliográficas}

Algert, S. J, Baameur, A., \& Renvall, M. J. (2014). Vegetable output and cost savings of community gardens in San Jose, California. Journal of the Academy of Nutrition and Dietetics 114, 10721076. https://doi.org/10.1016/j.jand.2014.02.030 Chipantiza-Masabanda, J. G., Bonilla-Bonilla, A. E., \& Jativa-Reyes, M. F. (2021). Huertos urbanos y periurbanos horizontales-verticales para el fomento de la educación ambiental sostenible. Formación universitaria, 14(2), 165-172.

https:// doi.org/10.4067/S071850062021000200165

Davcev, D., Mitreski, K., Trajkovic, S., Nikolovski, V., \& Koteli, N. (2018, June). IoT agriculture system based on LoRaWAN. In 2018 14th IEEE International Workshop on Factory Communication Systems (WFCS) (pp. 1-4). IEEE. https://doi.org/10.1109/WFCS.2018.8402368

Díaz, I. (2016). Granjas verticales: una respuesta sostenible al crecimiento urbano. Revista Prisma Tecnológico, 7(1), 3-6.

Hernández, R. A. (2020). Covid-19 y América Latina y el Caribe: los efectos económicos diferenciales en la región. Documentos de Trabajo (IAES, Instituto Universitario de Análisis Económico y Social) (6), 1-50.

Ji, M., Yoon, J., Choo, J., Jang, M., \& Smith, A.
(2019, March). LoRa-based Visual Monitoring Scheme for Agriculture IoT. In 2019 IEEE Sensors Applications Symposium (SAS) (pp. 1-6). IEEE. https://doi.org/10.1109/SAS.2019.8706100 Kodali, R. K., Yerroju, S., \& Sahu, S. (2018, August). Smart farm monitoring using LoRa enabled IoT. In Proc. 2nd Int. Conf. Green Comput. Internet Things (ICGCIoT).

https://doi.org/10.1109/ICGCIoT.2018.8753086 López, J. E. G., Chavez, J. C., \& Sánchez, A. K. J. (2017, March). Modelado de una red de sensores y actuadores inalámbrica para aplicaciones en agricultura de precisión. In 2017 IEEE Mexican Humanitarian Technology Conference (MHTC) (pp. 109-116). IEEE.

https://doi.org/10.1109/MHTC.2017.7926210

Martínez, J., y Pérez, D. E. (2017). Desarrollo de un sistema de control para el riego y la iluminación en una granja vertical (Tesis de pregrado). Universidad Autónoma de Occidente.

Orozco, O. L., Mendoza, M. G., Santoyo, J. O., \& Gamboa, A. G. (2018). Modelo de datos para el monitoreo de variables agro-climatológicas de huertos urbanos utilizando internet de las cosas y cómputo en la nube. Research in Computing Science, 147, 239-248.

https://doi.org/10.13053/rcs-147-8-18

Puengsungwan, S., \& Jiraserccamomkul, K. (2018, November). IoT Based Stress Detection for Organic Lettuce Farms Using Chlorophy11 Fluorescence (ChF). In 2018 Global Wireless Summit (GWS) (pp. 354-357). IEEE. https://doi. org/10.1109/GWS.2018.8686651

Ramírez, D. A. (2020). Automatización de sistema de riego para granjas verticales modulares.

Rincón Zapata, C. (2015). Sistema de monitoreo y control de huertos urbanos (Tesis de Pregrado), Uniandes. Facultad de Ingeniería. Departamento de Ingeniería Eléctrica y Electrónica. Bogotá Colombia.

Sánchez, J. G., \& Restrepo, J. V. (2018). Consideraciones de diseño en el prototipado de una maceta inteligente con sistema de irrigación autónomo gobernado por las ecuaciones de Penman-Moteith y Richards. Revista Tecnología Y Productividad, 4(4), 173-182.

https://doi.org/10.23850/24632465.2341 
Sanz-Cobeña, A., Puigdueta-Bartolomé, I., Larruscain, Á., Borrella, I., Álvarez, S., Cruz, J. L., ... \& Iglesias, A. (2017). Los huertos urbanos como herramienta de trasformación socio-agroalimentaria y medida de mitigación de GEI: el caso de Madrid. In Presented at the V Workshop Remedia.

Suciu, G., Istrate, C. I., \& Dițu, M. C. (2019, June). Secure smart agriculture monitoring technique through isolation. In 2019 Global IoT Summit (GIoTS) (pp. 1-5). IEEE.

https://doi.org/10.1109/GIOTS.2019.8766433

TEC, Granjas verticales, la nueva forma de cultivar. (2020). Recuperado de https://www.tec.ac. $\mathrm{cr} /$ pensis/articulos/granjas-verticales-nueva-forma-cultivar

Tsiatsis, V., Karnouskos, S., Holler, J., Boyle, D., \& Mulligan, C. (2018). Internet of Things Technologies and Applications for a New Age of Intelligence. (C. M. Vlasios Tsiatsis, Stamatis Karnouskos, Jan Höller, David Boyle, Ed.) (Second Edi). Academic Press.

Tzounis, A., Katsoulas, N., Bartzanas, T., \& Kittas, C. (2017). Internet of Things in agriculture, recent advances and future challenges. Biosystems Engineering, 164, 31-48.

https://doi.org/10.1016/j.biosys temseng.2017.09.007

Yoon, C., Huh, M., Kang, S. G., Park, J., \& Lee, C. (2018, February). Implement smart farm with IoT technology. In 2018 20th International Conference on Advanced Communication Technology (ICACT) (pp. 749-752). IEEE. https://doi. org/10.23919/ICACT.2018.8323907

Zuil Oliva, M. (2015). Integración de servicios de voz y Google Maps (Tesis de Pregrado). Universidad Carlos III de Madrid. Departamento de Informática. Ingeniería Técnica en Sonido e Imagen. http://hdl.handle.net/10016/25267 